\title{
Efficacy and Safety of Autologous Stromal Vascular Fraction in the Treatment of Empty Nose Syndrome
}

\author{
Do-Youn $\mathrm{Kim}^{1} \cdot$ Hye Ran Hong ${ }^{1} \cdot$ Eun Wook $\mathrm{Choi}^{2} \cdot$ Sang Won Yoon ${ }^{2}$ Yong Ju Jang ${ }^{1}$ \\ ${ }^{I}$ Department of Otolaryngology, Asan Medical Center, University of Ulsan College of Medicine, Seoul; \\ ${ }^{2}$ Prostemics Research Institute, Seoul, Korea
}

Objectives. Regenerative treatment using stem cells may serve as treatment option for empty nose syndrome (ENS), which is caused by the lack of turbinate tissue and deranged nervous system in the nasal cavity. We aimed to assess the efficacy and safety of the autologous stromal vascular fraction (SVF) in the treatment of ENS.

Methods. In this prospective observational clinical study, we enrolled 10 ENS patients who volunteered to undergo treatment of ENS through the injection of autologous SVF. Data, including demographic data, pre- and postoperative Sino-Nasal Outcome Test-25 (SNOT-25) scores, overall patient satisfaction, and postoperative complications, were prospectively collected. Nasal secretion was assessed using the polyurethane foam absorption method, and the levels of biological markers were analyzed in both ENS group and control group using enzyme-linked immunosorbent assay. The SVF extracted from abdominal fat was diluted and injected into both inferior turbinates.

Results. Among the 10 initial patients, one was excluded from the study. Subjective satisfaction was rated as "much improved" in two and "no change" in seven. Among the improved patients, the mean preinjection SNOT-25 score was 55.0 and the score at 6 months after injection was 19.5. However, the average SNOT-25 score of nine participants at 6 months after injection (mean \pm standard deviation, 62.4 \pm 35.8 ) did not differ significantly from the baseline SNOT25 score $(70.1 \pm 24.7, P>0.05$, respectively). Among the various inflammatory markers assessed, the levels of interleukin (IL)-1 $\beta$, IL-8, and calcitonin gene-related peptide were significantly higher in ENS patients. Compared with preinjection secretion level, the nasal secretions from SVF-treated patients showed decreased expressions of IL-1 $\beta$ and IL-8 after injection.

Conclusion. Although SVF treatment appears to decrease the inflammatory cytokine levels in the nasal mucosa, a single SVF injection was not effective in terms of symptom improvement and patient satisfaction. Further trials are needed to identify a more practical and useful regenerative treatment modality for patients with ENS.

Keywords. Empty Nose Syndrome; Stem Cells; Stromal Vascular Fraction; Sino-Nasal Outcome Test-25; Cytokines

\section{INTRODUCTION}

Empty nose syndrome (ENS) is characterized by dry nose, painful nasal breathing, nasal obstruction, crusting, and other debilitating nasal symptoms [1]. Patients with ENS have a history of

\footnotetext{
- Received November 24, 2017

Revised March 7, 2018

Accepted March 29, 2018

- Corresponding author: Yong Ju Jang

Department of Otolaryngology, Asan Medical Center, University of Ulsan College of Medicine, 88 Olympic-ro 43-gil, Songpa-gu, Seoul 05505, Korea Tel: +82-2-3010-3712, Fax: +82-2-489-2773

E-mail: jangyj@amc.seoul.kr
}

turbinate reduction procedures, including turbinectomy, turbinoplasty, and radiofrequency volume reduction [2]. Once patients develop this crippling disorder, they not only experience nasal symptoms but may also experience anxiety and depression that can lead to suicidal ideas in extreme cases [3].

ENS can be managed by conservative and surgical options. In a certain subgroup of patients with relatively mild symptoms, conservative treatments such as moisturization, intermittent plugging, and application of nasal ointment are occasionally effective [4,5]. In patients exhibiting marked loss of turbinate tissue along with severe symptoms, turbinate reconstruction using alloplastic implants, hyaluronic acid fillers, and autologous carti-

Copyright (C) 2018 by Korean Society of Otorhinolaryngology-Head and Neck Surgery.

This is an open-access article distributed under the terms of the Creative Commons Attribution Non-Commercial License (http://creativecommons.org/licenses/by-nc/4.0)

which permits unrestricted non-commercial use, distribution, and reproduction in any medium, provided the original work is properly cited. 
lage are reported as effective in alleviating symptoms and thereby improving the quality of life [6-8]. However, none of currently existing treatment modalities yield truly satisfactory treatment outcomes. In fact, several patients do not respond to the various conservative and surgical treatment options currently available [9]. Even in patients exhibiting improvement after surgical treatment, the extent of improvement represents a partial response, thus indicating the need for novel treatment modalities that supplement the effect of surgical reconstruction of the missing turbinate.

Nasal turbinates are an important functional organ that provides physical resistance to the inspired airflow, along with humidification and temperature regulation of the inspired air. In addition to this air-conditioning function, the epithelial layer and subepithelial lamina propria of the turbinates act as important innate defense organs [3,10]. Therefore, the loss of turbinate tissue may lead to derangement in airflow dynamics, alteration of the character of the inspired air, and damage to the innate defense mechanism of the nose [11]. Furthermore, surgical trauma to nerve endings during surgery may result in various ENS symptoms, such as painful breathing and inability to feel the inspired air. However, research on the pathophysiology of ENS remains inadequate, thus limiting our understanding and the appropriate management of this difficult condition.

Regenerative medicine using stem cells is gaining increasing applicability in various medical conditions. The basic premise of ENS is the loss of a functional nasal organ. Hence, stem cell treatments can be considered useful for regenerating the turbinate tissue and restoring its function. Moreover, the diagnosis of ENS has primarily depended on clinical history, until recently [5]. Thus, there is no available biologic marker of ENS that can be used to better understand the pathophysiology, obtain an accurate diagnosis, and follow up the surgical outcomes.

The stromal vascular fraction (SVF) from the autologous adipose tissue contains mesenchymal stem cells and other cellular components known to have regenerative and anti-inflammatory potential.Various studies have described the beneficial effects of

\section{H I G G H L I I G H T T S}

- The effect of autologous stromal vascular fraction injection was examined in nine patients with empty nose syndrome.

- The overall Sino-Nasal Outcome Test-25 score at 6 months after injection did not differ significantly from that of before the injection.

- The levels of interleukin (IL)-1 $\beta$, IL-8, and calcitonin generelated peptide were significantly higher in empty nose syndrome patients.

- Nasal secretions from stromal vascular fraction-treated patients showed decreased expressions of IL-1 $\beta$ and IL-8 after injection. treatment with SVF obtained from adipose tissue in the improvement of various medical conditions [12-16]. Therefore, to assess the therapeutic potential of SVF in the management of ENS, and to identify a biologic marker that can help better understand the pathophysiology of ENS, we conducted a prospective clinical observation study on a group of ENS patients from our institution.

\section{MATERIALS AND METHODS}

\section{Study population}

A total of 10 patients (seven males and three females; age, 22 to 55 years) with ENS who were referred to and diagnosed at Asan Medical Center, Seoul, Korea, were enrolled in this prospective observational clinical study. ENS was diagnosed based on the following criteria: (1) previous turbinate reduction surgery and positive cotton test [17]; (2) partially absent inferior turbinate tissue and abnormally wide nasal cavities, confirmed via endoscopic examinations and contrast-non-enhanced computed tomography scans; and (3) subjective symptoms, including paradoxical nasal obstruction, nasal dryness, facial or nasal pain on inspiration, nasal crusting or discharge, and headache [18]. Patients who underwent total inferior turbinectomy were excluded from the present study, as they were better suited for turbinate reconstruction using costal cartilage. All patients underwent preoperative psychological screening to exclude confounding psychiatric disorders. This study was approved by the Institutional Review Board of the Asan Medical Center (IRB No. 2015-0687), and informed consent was obtained from all the enrolled patients.

\section{Analysis of nasal secretion}

Nasal secretion analysis was performed according to the method described previously [19]. In brief, nasal secretions were collected using modified polyurethane sponge (Nasopore Standard, Groningen, The Netherlands), which was placed into each nasal cavity, posterior to the mucocutaneous junction, under direct visualization using endoscopy, and was left in place for $10 \mathrm{~min}$ utes. Following its removal, fluid was extracted from the sampler by centrifugation after the addition of isotonic saline solution. The levels of mucin-5AC (MUC5AC) and lactoferrin (LTF), as markers of glandular secretion; substance $\mathrm{P}$ and calcitonin generelated peptide (CGRP), as markers for neurogenic inflammation; and interleukin (IL)-1 $\beta$, IL-8, and tumor necrosis factor (TNF)- $\alpha$, as commonly used markers of nonspecific airway inflammation, were assessed. The concentrations of all substances were determined in duplicate and calculated as the actual concentrations in the undiluted specimen, as it had been obtained, by each sampling technique. The specimens were diluted to fit in the working range of the various assays. Nasal secretion collection was performed in each patient at 2 weeks before SVF injec- 
tion and 6 months after SVF injection. In order to compare the biological marker concentration of ENS patients with that of normal control, nasal secretion collection was also conducted in five healthy volunteers (control group). These five normal volunteers (three males and two females; age, 27 to 35 years) exhibited normal endoscopic findings, and had no history of sinonasal surgery, sinonasal abnormalities on computed tomography, or nasal symptoms.

\section{Preparation of SVF and injection}

The adipose tissue harvesting procedure was performed in the operating room with intravenous anesthesia with propofol (1.5 $\mathrm{mg} / \mathrm{kg}$ ). For free abdominal fat harvesting, a 1.5-2 cm transverse midline incision site was first outlined approximately $1 \mathrm{~cm}$ below the umbilicus. Thereafter, $1 \%$ lidocaine with 1:100,000 epinephrine was injected into the proposed surgical site. The \#10 blade was used to make an incision through the skin and subcutaneous tissue to expose the abdominal fat pad. After adequate tension was maintained on the fat graft, the fat was widely undermined, superficial to the rectus abdominis muscle fascia, to a similar extent as that for the overlying skin dissection. Approximately 50-100 g of adipose fat was extracted in a block shape from the lower abdomen, maintained in tissue culture media on ice, and washed with phosphate-buffered saline to remove the contaminating blood cells. Harvested tissue was immediately sent to the lab for further processing. The washed adipose tissue was enzymatically digested using collagenase $(0.075 \%)$ at $37^{\circ} \mathrm{C}$ for 45 minutes. The enzyme reaction was inactivated and centrifuged at 2,000 rpm for 10 minutes to obtain a high-density SVF pellet. The cell suspension was filtered through a $70 \mu \mathrm{m}$ nylon mesh to remove undispersed tissue elements and red blood cells. The obtained SVF was morphologically analyzed using an inverted microscope and the cell viability was verified by using trypan blue. This differentiated SVF was diluted to a concentration of $3 \times 10^{7}$ cells $/ \mathrm{mL}$ and $1.5 \mathrm{~mL}$ each was injected in most medial surface of both inferior turbinates. SVF isolation and in- jection were performed within 2 hours. To assess the association between SVF injection and the outcome, we did not prescribe any medication that could affect nasal secretion analysis during this study period.

\section{Outcome measurements}

All patients were evaluated in terms of the severity of the nasal symptoms using the Sino-Nasal Outcome Test-25 (SNOT-25) questionnaire as well as overall patient satisfaction. The SNOT25 questionnaire comprised 25 items that quantify the symptoms associated with ENS; each item is rated on a score from 0 (no symptom) to 5 (severe symptoms) [20]. We administered the SNOT-25 questionnaire to the enrolled patients at 2 weeks before SVF injection and at 2 weeks, 1 month, 2 months, 3 months, and 6 months after SVF injection.

\section{Statistical analyses}

Statistical analyses were performed using IBM SPSS ver. 21.0 (IBM Corp., Armonk, NY, USA). For quantitative variables, values are presented as mean \pm standard deviation. The Wilcoxon signed-rank test was used to compare outcomes before and after SVF injection. Differences were considered significant at $P<0.05$.

\section{RESULTS}

Ten patients (age, $35.8 \pm 17.7$ years; three females) received SVF injections in both inferior turbinates, and of these. One patient was excluded from the study during the follow-up period because he decided to drop out. The mean overall SNOT-25 score at 6 months after SVF injection $(62.4 \pm 35.8)$ did not differ significantly with that before the injection $(70.1 \pm 24.7, P>0.05$, respectively). Analysis of the subjective outcome indicated that two patients $(22 \%)$ were satisfied with their improved outcome, and that seven patients (78\%) felt no change after SVF injec-

Table 1. Demographics, SNOT-25 scores, patient satisfaction, and complications in patients with empty nose syndrome

\begin{tabular}{|c|c|c|c|c|c|c|c|}
\hline Case no. & Age (yr) & Sex & $\begin{array}{l}\text { Previous } \\
\text { treatment }\end{array}$ & $\begin{array}{c}\text { SNOT-25 } \\
\text { (preinjection) }\end{array}$ & $\begin{array}{c}\text { SNOT-25 } \\
\text { (6 months postinjection) }\end{array}$ & $\begin{array}{l}\text { Overall subjective } \\
\text { improvement }\end{array}$ & Complication \\
\hline 1 & 20 & Male & RFA & 75 & 38 & Improved & $x$ \\
\hline 2 & 37 & Female & SMR & 80 & 116 & No change & $x$ \\
\hline 3 & 47 & Male & SMR & 35 & 1 & Improved & $x$ \\
\hline 4 & 45 & Male & SMR & 74 & 58 & No change & $x$ \\
\hline 5 & 30 & Male & RFA & 33 & 29 & No change & $x$ \\
\hline 6 & 22 & Male & PT & 99 & 102 & No change & $\times$ \\
\hline 7 & 29 & Female & RFA & 71 & 76 & No change & Seroma \\
\hline 8 & 55 & Male & $\mathrm{RFA}+\mathrm{SMR}$ & 101 & 94 & No change & x \\
\hline 9 & 38 & Female & RFA & 63 & 48 & No change & $x$ \\
\hline 10 & 47 & Male & SMR & - & - & Drop out & - \\
\hline Mean $\pm S D$ & $35.8 \pm 11.7$ & & & $70.1 \pm 24.7$ & $62.4 \pm 35.8$ & & \\
\hline
\end{tabular}

SNOT-25, Sino-Nasal Outcome Test-25; RFA, radiofrequency ablation; SMR, submucosal resection; PT, partial turbinectomy; SD, standard deviation. 

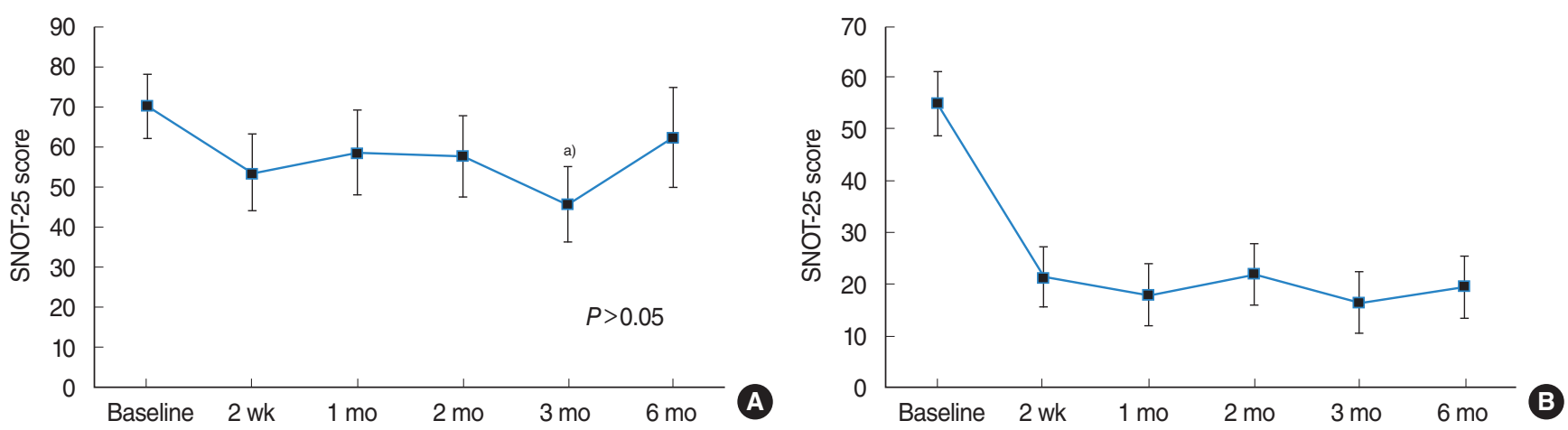

Fig. 1. Sino-Nasal Outcome Test-25 (SNOT-25) scores at different time periods after stromal vascular fraction (SVF) injection. The mean SNOT25 scores ranged from 0 to 125, wherein a higher score indicated greater empty nose syndrome severity. (A) The data of nine patients undergoing SVF injection are included, and the mean baseline SNOT-25 score was 70.1 \pm 24.7 . The overall change was not consistent over time $(P>0.05)$. The mean SNOT-25 score was $45.78 \pm 27.74$ at 3 months after injection as compared to the baseline score. (B) The data of two improved patients are also included, who had a mean baseline SNOT-25 score of 55.0 \pm 20.0 . The effect of SVF injection was evident after 2 weeks, and was maintained thereafter. a) $P=0.009$ vs. baseline.

tion. Among the improved patients, the mean preinjection SNOT-25 score was 55.0 and the score at 6 months after the injection was 19.5 (Table 1 ).

To identify the outcome in a time-ordered sequence, we analyzed the SNOT-25 scores for these patients. The changes in the overall SNOT-25 scores were not significant over time, until the 6-month follow-up $(P>0.05)$. However, the mean improvement in the SNOT-25 score was significantly different $(95 \%$ confidence interval, 7.86 to $40.81 ; P=0.009$ ) only at 3 months after injection, as compared to the preinjection baseline score. The mean SNOT-25 score of two patients who were satisfied with the result indicated significant improvement at 2 weeks after SVF injection and the effect was maintained thereafter (Fig. 1).

To determine a suitable biological marker of ENS, multiple markers for inflammation were selected and evaluated in this study. Among the various inflammatory markers assessed, the levels of IL-1 $\beta$, IL-8, and CGRP were significantly higher in the ENS group than in the control group $(P<0.001)$. However, the levels of LTF did not significantly differ between the ENS and control group. The other markers, including TNF- $\alpha$, MUC5AC, and substance $\mathrm{P}$, were undetectable in the ENS and control group. Compared with the nasal secretions before the injection, nasal secretions from SVF-treated patients showed decreased levels of IL-1 $\beta$, IL-8, and CGRP after SVF injection. Among these biological markers, the concentrations of IL- $1 \beta$ and IL- 8 were significantly decreased after SVF injection $(P<0.005)$. Although the concentration of CGRP and LTF was decreased after SVF injection, no significant decrease was found in the levels of those biomarkers (Fig. 2).

There were no recipient site complications, such as hemorrhage, pain, or embolism. However, complications were observed in the form of a seroma in one patient, who was treated via ultrasound-guided aspiration during the follow-up period.

\section{DISCUSSION}

Several recent studies in humans and animals have repeatedly shown that autologous adipose derived stem cell (ADSC) and SVF have regenerative and anti-inflammatory potential $[13,15$, 21-23]. Regenerative medicine-based treatments, including platelet-rich plasma, platelet-rich lipotransfer, ADSC, SVF, extracellular matrix, and fat graft, may be suitable alternatives for the treatment of ENS. The benefits of this treatment include easy access, minor tissue damage after transplantation, rapid proliferation, and wide range of source. However, only few previous reports have described the use of stem cells for the treatment of ENS $[23,24]$. A previous study reported the effect of ADSC on the nasal mucosa and the underlying mechanism in the treatment of ENS [23]; the authors used fat particles combined with ADSC in 30 patients with ENS. The results of nasal endoscopy and mucociliary clearance assessment were significantly improved, and inflammation was significantly reduced in all the patients. The authors concluded that ADSC transplantation may be effective for improving symptoms and mucosal function in patients with ENS. In that study, the authors performed three consecutive ADSC injections to areas of damaged nasal mucosa and inferior turbinates at 10-day intervals. The ADSC developed a polygonal cobblestone shape, characteristic of human epithelial cells. The author concluded that ADSCs improved the function of epithelial cells by upregulating the expression of specific epithelial markers, such as cytokeratin 7, 14, and 19 , which consequently improved the nasal mucosa function in patients with ENS.

Stem cells are characterized by their properties of self-renewal and multipotency. Both ADSCs and SVF include mesenchymal stem cells that can differentiate into multiple lineages of mature cells. SVF is the cell population that is obtained from fat tissue after a mini-lipoaspirated procedure. The SVF contains 
IL-1 $\beta$

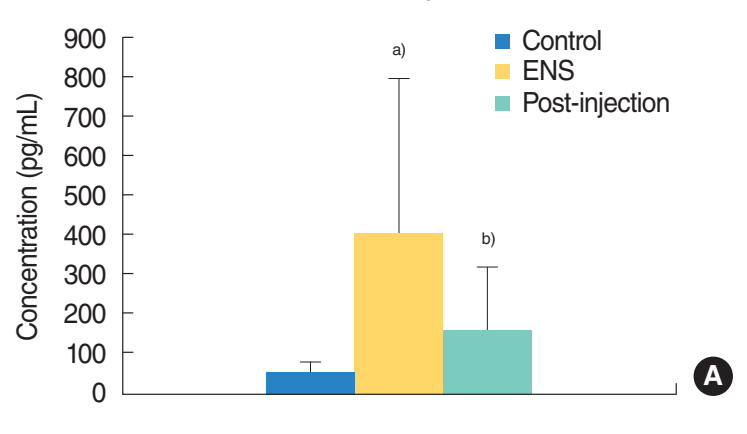

CGRP

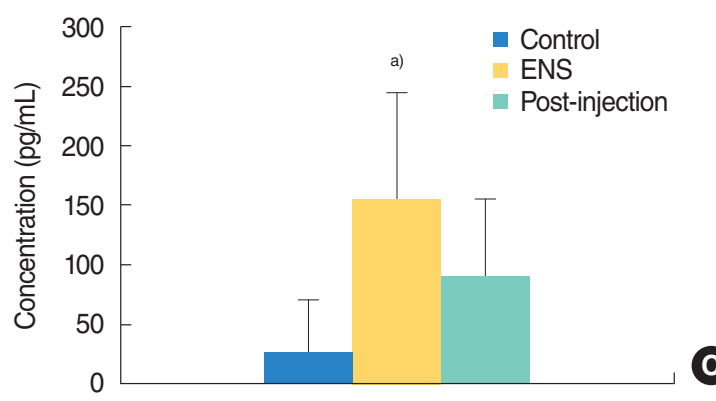

IL-8

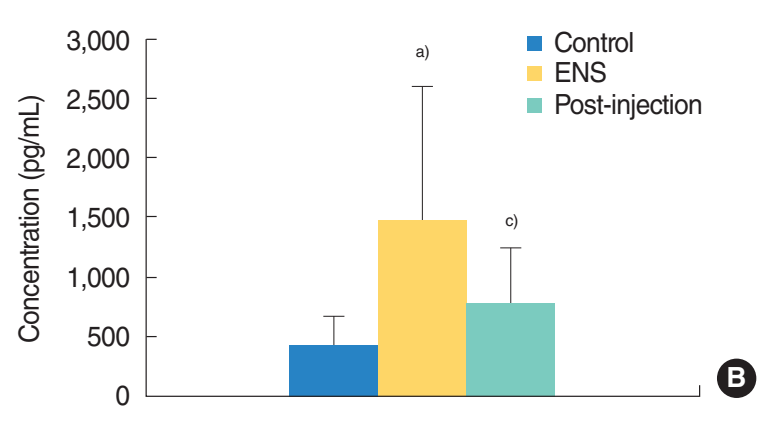

LTF

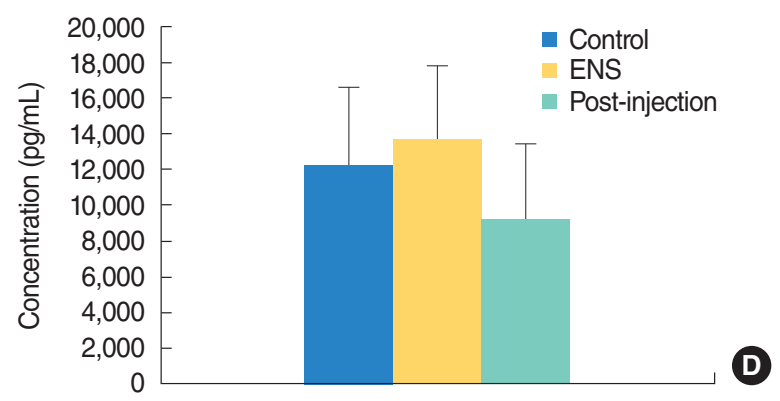

Fig. 2. Concentration of biomarkers of the control group, empty nose syndrome (ENS) group, and after stromal vascular fraction (SVF) injection group. (A) Comparisons of interleukin (IL)-1B, (B) IL-8, (C) calcitonin gene-related peptide (CGRP), (D) lactoferrin (LTF) levels. The IL-1B, IL-8, and CGRP levels were significantly higher in the ENS group than in the control group. The levels of IL-1B and IL-8 were significantly decreased after SVF injection. The LTF level did not significantly differ between the ENS and control group. The levels of CGRP and LTF were not significantly decreased after SVF injection. ${ }^{a)} P<0.01$ vs. the control group. ${ }^{\text {b) }} P=0.013$ vs. ENS group. ${ }^{c)} P=0.014$ vs. ENS group.

various types of cells, including stem cells, hematopoietic cells, immune cells, smooth muscle cells, endothelial cells, and other cell components, and markedly differs from ADSCs $[13,25]$. The SVF has a similar regenerative potential as ADSCs, but can be purified at one-tenth the price of stem cell therapy, which makes it a valuable and effective treatment for restoring damaged tissue and reducing inflammation. The SVF is an attractive therapeutic method given that the harvesting process is safe and the cells are readily available in large quantities. Accordingly, the SVF has been found to be effective in treating conditions from various medical fields including osteoarthritis, degenerative disc disease, sclerosis, tendinopathy, congestive heart failure, chronic obstructive pulmonary disease, radiation necrosis, xerostomia, and multiple sclerosis [12-16]. The entire process of obtaining SVF can be performed within 60-90 minutes in a clean facility; however, the complete isolation of a homogenous population of ADSCs from SVF requires 24 hours in a cell culture facility. Thus, the disadvantages of ADSCs include that they require a considerable amount of time and substantial expense to obtain $[13,25]$. Hence, the SVF can be used in these patients, which would obviate the need for a cell culture procedure. In the present study, the isolation and preparation of SVF was conducted by a company (Prostemics, Seoul, Korea) located within our hospital campus. The SVF from adipose tissue enabled us to col- lect as much tissue as possible and obtain a sufficient number of stem cells to achieve therapeutic potency. Flow cytometry could not perform on a portion of all obtained SVF pellet in terms of time limit. However, flow cytometry was sometimes conducted in case of remained sample and SVF was maintained proportion of mesenchymal stem cell about $40 \%$ by standard protocol and expert researchers. Therefore, we performed single adipose SVF injection in patients with ENS. In the present clinical trial, although two of nine patients showed improvement, we failed to confirm the significantly beneficial effect of adipose SVF in the treatment of ENS in most of our patients.

There may be some explanation for the lack of favorable effect on ENS with the use of adipose SVF. A single application of adipose SVF may not be sufficient to achieve the desired therapeutic effect for this chronically debilitating illness. However, due to the harvesting-related morbidity, repeated harvesting of adipose SVF is practically burdensome to both patients and clinicians. This is a serious limitation of our choice of adipose SVF. Hence, the use of commercially available, easy-to-acquire cultured stem cells may serve as an alternative option, which needs to be explored in further studies.

In the present study, we could identify several candidate biomarkers for ENS. The pathophysiologic mechanism of ENS involves a complicated signal pathway and various biologic mole- 
cules. The factors contributing to ENS include: alteration of the airflow pattern; reduced mucosal area; decreased humidification and increased warming; reduced nasal airflow resistance; loss of sensory, tactile, and thermal receptors needed for inhaled air treatment; and neural damage [2,26]. The levels of biological markers in nasal secretions can provide valuable information regarding nasal pathophysiology. In the present study, we selected IL-1 $\beta$, IL-8, TNF- $\alpha$, MUC5AC, LTF, substance P, and CGRP as candidate markers. Proinflammatory cytokines, such as TNF- $\alpha$, IL-1 $\beta$, and IL- 8 , are potent multifunctional cytokines that play an important role in the pathogenesis of many inflammatory diseases [27]. MUC5AC and LTF are markers of glandular secretion of the nasal mucosa $[28,29]$. Neuropeptides, such as CGRP and substance P, mediate neurogenic inflammation [30]. In the present study, nasal secretion analysis in the ENS group showed an increased level of cytokines such as IL-1 $\beta$, IL-8, and CGRP, in comparison with the normal control group. These findings may suggest that ENS is a chronic inflammatory condition caused by altered airflow, which offers novel insights on the pathophysiology of ENS.

Nasal secretion analysis is commonly performed by nasal lavage; however, it is difficult to obtain consistently good results using the conventional nasal lavage technique. Therefore, in the present study, we used a modified polyurethane sponge for secretion collection and analysis, which is considered to be superior to the conventional nasal lavage technique. A previous study described the analysis of nasal concentrations using the nasal lavage technique, nasal spray-blow technique, filter paper method, and polyurethane sponge sampler technique; the authors indicated that the nasal lavage technique was approximately 10 times less effective than the modified polyurethane sponge method [19]. Moreover, due to the unpredictable dilution obtained by the nasal lavage technique, the analyzed concentrations may be below the detection limit of high sensitivity assays. In the present study, the use of nasal secretion analysis with modified polyurethane sponge rarely yielded concentrations below the detection limit.

A single injection of SVF did not show successful outcomes in terms of symptom improvement and patient satisfaction. However, our findings regarding the expression of inflammatory cytokines in ENS patients suggest that SVF injection reduces the levels of inflammatory cytokines, including IL-1 $\beta$ and IL-8, in the nasal mucosa. In the two patients who exhibited symptom improvement after SVF injection, we could not determine the reason underlying the beneficial effect of SVF. The improvements could be due to the regeneration of the turbinate tissue, but may be most likely due to the improvement in inflammation by the SVF, as demonstrated by changes in the cytokine profile. The potential anti-inflammatory role of the SVF has been wellrecognized in previous studies, but needs to be further assessed, particularly in cases of sinonasal inflammation.

The present study had certain limitations of note. First, the number of patients was limited; in particular, we only enrolled five volunteers as normal controls for nasal secretion analysis. Second, the histologic change caused by stem cell injection was not examined, as we were deeply concerned regarding the potential adverse effects of mucosal biopsy on our patients, whose problem was caused by the prior surgical procedure on the turbinates.

SVF injection appears to reduce the inflammatory cytokine levels in the nasal mucosa, a single injection of SVF did not bring out uniformly successful outcome in terms of improvement of symptoms and patient satisfaction. Further studies are necessary to find out more practical and effective regenerative treatment modality in patient with ENS.

\section{CONFLICT OF INTEREST}

This study was supported by Prostemics Co., LTD., Seoul, Korea. The funder had no role in the design, collection, analysis or interpretation of this study. No other potential conflict of interest relevant to this article was reported.

\section{ACKNOWLEDGMENTS}

The authors are grateful to Prostemics Co., LTD., Seoul, Korea, for funding this research work.

\section{REFERENCES}

1. Oluwole M, Mills RP. An audit of the early complications of turbinectomy. Ann R Coll Surg Engl. 1994 Sep;76(5):339-41.

2. Houser SM. Surgical treatment for empty nose syndrome. Arch Otolaryngol Head Neck Surg. 2007 Sep;133(9):858-63.

3. Houser SM. Empty nose syndrome associated with middle turbinate resection. Otolaryngol Head Neck Surg. 2006 Dec;135(6):972-3.

4. Kuan EC, Suh JD, Wang MB. Empty nose syndrome. Curr Allergy Asthma Rep. 2015 Jan;15(1):493.

5. Shah K, Guarderas J, Krishnaswamy G. Empty nose syndrome and atrophic rhinitis. Ann Allergy Asthma Immunol. 2016 Sep;117(3): 217-20.

6. Jung JH, Baguindali MA, Park JT, Jang YJ. Costal cartilage is a superior implant material than conchal cartilage in the treatment of empty nose syndrome. Otolaryngol Head Neck Surg. 2013 Sep;149(3): 500-5.

7. Modrzynski M. Hyaluronic acid gel in the treatment of empty nose syndrome. Am J Rhinol Allergy. 2011 Mar-Apr;25(2):103-6.

8. Saafan ME. Acellular dermal (alloderm) grafts versus silastic sheets implants for management of empty nose syndrome. Eur Arch Otorhinolaryngol. 2013 Feb;270(2):527-33.

9. Leong SC. The clinical efficacy of surgical interventions for empty nose syndrome: a systematic review. Laryngoscope. 2015 Jul;125(7): 1557-62.

10. Coste A, Dessi P, Serrano E. Empty nose syndrome. Eur Ann Otorhinolaryngol Head Neck Dis. 2012 Apr;129(2):93-7.

11. Dayal A, Rhee JS, Garcia GJ. Impact of middle versus inferior total 
turbinectomy on nasal aerodynamics. Otolaryngol Head Neck Surg. 2016 Sep;155(3):518-25.

12. Kang SK, Shin MJ, Jung JS, Kim YG, Kim CH. Autologous adipose tissue-derived stromal cells for treatment of spinal cord injury. Stem Cells Dev. 2006 Aug;15(4):583-94.

13. Kim DY, Ji YH, Kim DW, Dhong ES, Yoon ES. Effects of platelet-rich plasma, adipose-derived stem cells, and stromal vascular fraction on the survival of human transplanted adipose tissue. J Korean Med Sci. 2014 Nov;29 Suppl 3:S193-200.

14. Lataillade JJ, Doucet C, Bey E, Carsin H, Huet C, Clairand I, et al. New approach to radiation burn treatment by dosimetry-guided surgery combined with autologous mesenchymal stem cell therapy. Regen Med. 2007 Sep;2(5):785-94.

15. Premaratne GU, Ma LP, Fujita M, Lin X, Bollano E, Fu M. Stromal vascular fraction transplantation as an alternative therapy for ischemic heart failure: anti-inflammatory role. J Cardiothorac Surg. 2011 Mar;6:43.

16. Rodriguez JP, Murphy MP, Hong S, Madrigal M, March KL, Minev B, et al. Autologous stromal vascular fraction therapy for rheumatoid arthritis: rationale and clinical safety. Int Arch Med. 2012 Feb;5:5.

17. Thamboo A, Velasquez N, Habib AR, Zarabanda D, Paknezhad H, Nayak JV. Defining surgical criteria for empty nose syndrome: validation of the office-based cotton test and clinical interpretability of the validated Empty Nose Syndrome 6-Item Questionnaire. Laryngoscope. 2017 Aug;127(8):1746-52.

18. Jang YJ, Kim JH, Song HY. Empty nose syndrome: radiologic findings and treatment outcomes of endonasal microplasty using cartilage implants. Laryngoscope. 2011 Jun;121(6):1308-12.

19. Riechelmann H, Deutschle T, Friemel E, Gross HJ, Bachem M. Biological markers in nasal secretions. Eur Respir J. 2003 Apr;21(4): 600-5.

20. Jiang C, Wong F, Chen K, Shi R. Assessment of surgical results in patients with empty nose syndrome using the 25-item Sino-Nasal Outcome Test Evaluation. JAMA Otolaryngol Head Neck Surg. 2014 May;140(5):453-8.

21. Palumbo P, Miconi G, Cinque B, La Torre C, Lombardi F, Zoccali G, et al. In vitro evaluation of different methods of handling human liposuction aspirate and their effect on adipocytes and adipose derived stem cells. J Cell Physiol. 2015 Aug;230(8):1974-81.

22. Phipps KD, Gebremeskel S, Gillis J, Hong P, Johnston B, Bezuhly M. Alternatively activated M2 macrophages improve autologous fat graft survival in a mouse model through induction of angiogenesis. Plast Reconstr Surg. 2015 Jan;135(1):140-9.

23. Xu X, Li L, Wang C, Liu Y, Chen C, Yan J, et al. The expansion of autologous adipose-derived stem cells in vitro for the functional reconstruction of nasal mucosal tissue. Cell Biosci. 2015 Sep;5:54.

24. Friji MT, Gopalakrishnan S, Verma SK, Parida PK, Mohapatra DP. New regenerative approach to atrophic rhinitis using autologous lipoaspirate transfer and platelet-rich plasma in five patients: our experience. Clin Otolaryngol. 2014 Oct;39(5):289-92.

25. Tan SS, Loh W. The utility of adipose-derived stem cells and stromal vascular fraction for oncologic soft tissue reconstruction: is it safe? A matter for debate. Surgeon. 2017 Aug;15(4):186-9.

26. Scheithauer MO. Surgery of the turbinates and "empty nose" syndrome. GMS Curr Top Otorhinolaryngol Head Neck Surg. 2010;9: Doc03.

27. Tomassen P, Vandeplas G, Van Zele T, Cardell LO, Arebro J, Olze H, et al. Inflammatory endotypes of chronic rhinosinusitis based on cluster analysis of biomarkers. J Allergy Clin Immunol. 2016 May; 137(5):1449-56.e4.

28. Luo Q, Zhang Z, Liu D, Feng K, Jin X, Zhang J. Human neutrophil elastase induces MUC5AC overexpression in chronic rhinosinusitis through tumour necrosis factor-alpha converting enzyme. Acta Otolaryngol. 2016 Jun;136(6):641-8.

29. Raphael GD, Jeney EV, Baraniuk JN, Kim I, Meredith SD, Kaliner MA. Pathophysiology of rhinitis: lactoferrin and lysozyme in nasal secretions. J Clin Invest. 1989 Nov;84(5):1528-35.

30. Hauser-Kronberger C, Hacker GW, Franz P, Albegger K, Dietze O. CGRP and substance $P$ in intraepithelial neuronal structures of the human upper respiratory system. Regul Pept. 1997 Oct;72(2-3):7985. 